\title{
Sum Capacity for Single-Cell Multi-User Systems with $M$-Ary Inputs
}

\author{
Pei Yang ${ }^{1, *(1)}$, Yue $\mathrm{Wu}^{2}$, Liqiang Jin ${ }^{1}$ and Hongwen Yang ${ }^{1}$ \\ 1 School of Wireless Communication Center, Beijing University of Posts and Telecommunications, \\ Beijing 100876, China; jliq@bupt.edu.cn (L.J.); yanghong@bupt.edu.cn (H.Y.) \\ 2 School of Department of Electronic and Information Engineering, Anhui University of Finance and Economics, \\ Anhui 233030, China; wuyue233@126.com \\ * Correspondence: yp@bupt.edu.cn; Tel.: +86-010-6228-3233
}

Received: 30 June 2017; Accepted: 12 September 2017; Published: 15 September 2017

\begin{abstract}
This paper investigates the sum capacity of a single-cell multi-user system under the constraint that the transmitted signal is adopted from $M$-ary two-dimensional constellation with equal probability for both uplink, i.e., multiple access channel (MAC), and downlink, i.e., broadcast channel (BC) scenarios. Based on the successive interference cancellation (SIC) and the entropy power Gaussian approximation, it is shown that both the multi-user MAC and BC can be approximated to a bank of parallel channels with the channel gains being modified by an extra attenuate factor that equals to the negative exponential of the capacity of interfering users. With this result, the capacity of MAC and BC with arbitrary number of users and arbitrary constellations can be easily calculated which in sharp contrast with using traditional Monte Carlo simulation that the calculating amount increases exponentially with the increase of the number of users. Further, the sum capacity of multi-user under different power allocation strategies including equal power allocation, equal capacity power allocation and maximum capacity power allocation is also investigated. For the equal capacity power allocation, a recursive relation for the solution of power allocation is derived. For the maximum capacity power allocation, the necessary condition for optimal power allocation is obtained and an optimal algorithm for the power allocation optimization problem is proposed based on the necessary condition.
\end{abstract}

Keywords: sum capacity; power allocation; $M$-ary constellation; entropy power; multiple access channel; broadcast channel

\section{Introduction}

Recent years, with the rapid growth of mobile data traffic, a great deal of attentions have been attracted on the information theoretic issues of multi-user systems. When a base station receives data from its wireless terminals, multiple transmitters communicate simultaneously to a common receiver. Such many-to-one wireless communication scenarios, for which the common mathematical model is the Gaussian multiple-access channel (MAC), or in turn, the broadcast channel (BC), have been studied intensively in the literature [1-13]. It was pointed out Gaussian MAC and BC are dual [1], with which the capacity region and the transmitting strategy for MAC can be achieved from BC [2] and vice versa. In addition, the capacity region for Gaussian MAC or BC can be achieved through superposition coding and successive interference cancellation (SIC) $[3,4]$ while the maximum sum capacity can be obtained when all the power is allocated to the user with the best channel gain for Gaussian BC which is also true for Gaussian MAC by the duality. Although the power allocation can be greatly simplified with Gaussian BC [5-7] and MAC [8-10], they can never be realized in practice. Rather, the inputs must usually be drawn from discrete constellations with limited peak-to-average ratios (e.g., $M$-QAM, M-PSK) which is significantly depart from Gaussian distribution. Yet, no solutions 
has been found for calculating the sum capacity and allocating power that maximizes the sum capacity with non-Gaussian inputs.

As we know, no literature had been concerned on the study of sum capacity and power allocation for multi user situation due to the complexity for discrete input scenarios. For non-Gaussian inputs, there are no theoretical methods for calculating sum capacity of the multiuser AWGN channel. Even for the simplest single user scenario, there exists no closed form formula which can be used to calculate the capacity. The bounds and approximations for the capacity of point-to-point communication can be found in [14-17] which simplified the complicated integration into a closed-form solution. Usually, numerical solutions are available for estimating the capacity of a single user, e.g., Monte Carlo integration [18]. However, for multi user systems, it is too cumbersome to use Monte Carlo integration for estimating the sum capacity when the number of users is pretty large due to a series of mixed gaussian integration involved, let alone power allocation.

In this paper, first an approximation for the capacity of point-to-point communication with arbitrary inputs (e.g., QAM, PSK) is introduced, which is simple enough for calculation with high accuracy. Through the parallelization of MAC and BC based on the SIC and the concept of entropy power [19], a universal approximation for multi-user systems with arbitrary inputs distributions is obtained. Then an optimal algorithm that maximizes the sum capacity and a recursive relation for the solution of equal capacity power allocation is proposed based on the parallelization of MAC and BC.

\section{System Model}

\subsection{Multiple Assess Channel}

The system model for MAC is shown in Figure 1 where $K$ users transmit data simultaneously to a common base station. User $i$ encodes its data with a capacity achievable code of rate $R_{i}$ into signal $X_{i}$ satisfying $\mathbb{E}\left[\left|X_{i}\right|^{2}\right]=1$ and then, send $X_{i}$ with power $P_{i}$ through the uplink channel of gain $g_{i}$. The overall signal received by the base station can be expressed as

$$
Y=\sum_{i=1}^{K} \sqrt{P_{i} g_{i}} X_{i}+Z
$$

where $Z \sim \mathcal{C N}(0,1)$ is the complex Gaussian noise. Without loss of generality, assume that users are indexed in an ascending order of power gain, i.e., $0<g_{1} \leq g_{2} \leq \cdots \leq g_{K}$.

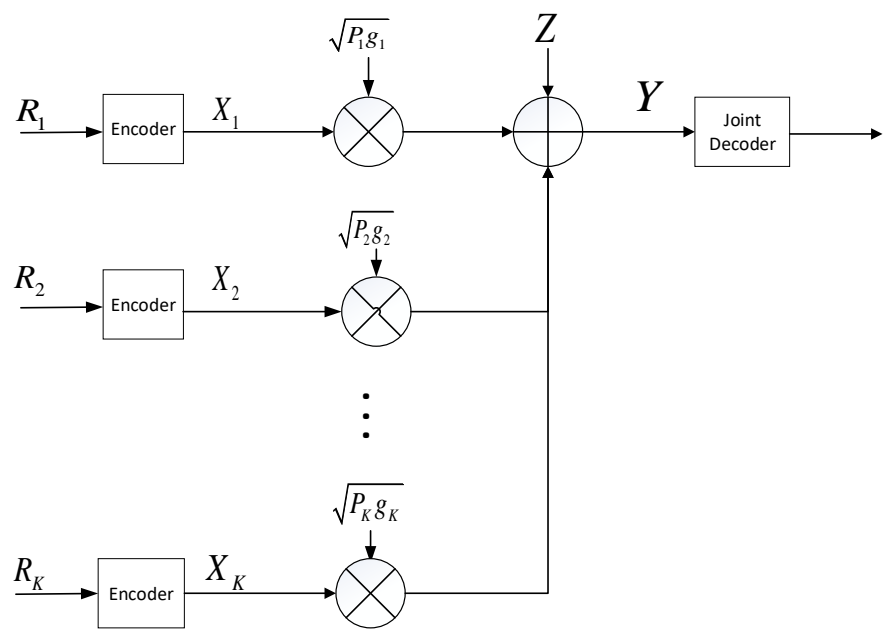

Figure 1. System model for a multi-user MAC system. 
The sum-capacity $C_{\text {sum }}$ is the maximum achievable sum rate $R_{1}+R_{2}+\cdots+R_{K}$, and is given by

$$
C_{\text {sum }}=\max \left\{I\left(Y ; X_{K} X_{K-1} \cdots X_{1}\right)\right\}
$$

where the maximization is over the joint distribution of $X_{1}, X_{2}, \cdots, X_{K}$.

For Gaussian encoders, $X_{1}, X_{2}, \cdots, X_{K}$ are independent and identically distributed (i.i.d.) $\mathcal{C N}(0,1)$. In this case, the sum capacity for a given power allocation $P_{1}, P_{2}, \cdots, P_{K}$ is $[2,3]$

$$
C_{\text {sum }}^{\text {Gaussian }}=\ln \left(1+\sum_{i=1}^{K} P_{i} g_{i}\right)
$$

Under the total power constraint $P_{1}+P_{2}+\cdots+P_{K}=P$, the sum-capacity can be maximized to

$$
C_{\text {sum }}^{\text {Gaussian }}=\ln \left(1+P g_{K}\right) \text {, }
$$

since $\sum_{i=1}^{K} P_{i} g_{i} \leq \sum_{i=1}^{K} P_{i} g_{K}=P g_{K}$. The optimal power allocation for the maximum sum capacity for Gaussian MAC is to allocate all power only to the strongest channel.

For discrete $M$-ary channel inputs, $X_{1}, X_{2}, \cdots, X_{K}$ are i.i.d. random variables taking values $\Omega=\left\{\alpha_{1}, \alpha_{2}, \cdots, \alpha_{M}\right\} \subset \mathbb{C}$ with equal probabilities. The sum capacity is given by

$$
\begin{aligned}
C_{\text {sum }} & =h(Y)-h(Y \mid X) \\
& =-\mathbb{E}[p(y)]-h(Z) \\
& =-\int_{-\infty}^{\infty} p(y) \ln p(y) \mathrm{d} y-\ln (2 \pi \mathrm{e}),
\end{aligned}
$$

where the probability density function (PDF) of the received signal $Y$ is

$$
p(y)=\sum_{i_{1}=1}^{M} \sum_{i_{2}=1}^{M} \cdots \sum_{i_{K}=1}^{M} \frac{1}{2 \pi M^{K}} \mathrm{e}^{-0.5\left|y-\sum_{j=1}^{K} \sqrt{P_{j} g_{j}} \alpha_{j_{j}}\right|^{2}} .
$$

and $M$ is the number of the discrete points of $\Omega$ for each user.

For point-to-point communication, i.e., $K=1$, the PDF of the received signal (6) becomes

$$
p(y)=\sum_{i=1}^{M} \frac{1}{2 \pi M} \mathrm{e}^{-0.5\left|y-\sqrt{P g} \alpha_{i}\right|^{2}} .
$$

Then the capacity of point-to-point communication can be denoted as

$$
C_{0}(\gamma)=-\int_{-\infty}^{\infty}\left(\sum_{i=1}^{M} \frac{1}{2 \pi M} \mathrm{e}^{-0.5\left|y-\sqrt{\operatorname{Pg}} \alpha_{i}\right|^{2}}\right) \log \left(\sum_{i=1}^{M} \frac{1}{2 \pi M} \mathrm{e}^{-0.5\left|y-\sqrt{\operatorname{Pg}} \alpha_{i}\right|^{2}} \mathrm{~d} y\right)-\ln (2 \pi \mathrm{e}) .
$$

The integration involved in (8) has no closed-form expression. There exists a simple approximation as [17]

$$
C_{0}(\gamma) \approx \log M\left(1-\sum_{i=1}^{N} a_{i} \mathrm{e}^{-b_{i} \gamma}\right)
$$

where $\gamma$ is the SNR, $a_{i}, b_{i}, N$ are the curve fitting parameters. The optimal parameters for QPSK, 8PSK, 16QAM constellations are given in Table 1. 
Table 1. Approximation parameters of (9) for QPSK, 8PSK, 16QAM modulations.

\begin{tabular}{cccc}
\hline Modulation & $\boldsymbol{a}$ & $\boldsymbol{b}$ & $\boldsymbol{N}$ \\
\hline QPSK & 1 & 0.6507 & 1 \\
\hline \multirow{2}{*}{ 8PSK } & 0.6130 & 0.1681 & \multirow{2}{*}{ 2 } \\
& 0.3855 & 0.8992 & \\
\hline \multirow{2}{*}{ 16QAM } & 0.7177 & 0.1225 & 2 \\
& 0.2804 & 0.8702 & \\
\hline
\end{tabular}

The calculation of (5) is more complicated than (8) due to the $M^{K}$ mixes of Gaussian distribution involved. The Monte Carlo integration which can be used for calculating (8) may not be used for calculating (5) especially when $K$ and $M$ are large. For example, for a MAC system with $K=10$ users and QPSK modulation $(M=4)$, there are $4^{10}$ items in the summation of (6). This problem will be addressed in next section.

To realize the sum capacity $C_{\text {sum }}$, a joint maximum likelihood (ML) decoding is required for the base station to jointly detect the codewords sent by all users. Alternatively, with the aid of SIC, $C_{\text {sum }}$ can also be realized with per user ML decoders. This is because that, according to the chain rule of mutual information [4], the mutual information $I\left(Y ; X_{K} X_{K-1} \cdots X_{1}\right)$ can be expressed as

$$
I\left(Y ; X_{K} X_{K-1} \cdots X_{1}\right)=I\left(Y ; X_{K}\right)+I\left(Y ; X_{K-1} \mid X_{K}\right)+\cdots+I\left(Y ; X_{1} \mid X_{K} \cdots X_{2}\right),
$$

which indicates that the receiver could first decode the data $X_{K}$ of user $K$, treating $X_{1}, X_{2}, \cdots, X_{K-1}$ as interferences, then subtract the signal $X_{K}$ from the receive signal $Y$ and then, decode the data $X_{K-1}$ of user $K-1$, and so on.

Note that (10) holds for arbitrary permutation of $X_{1}, X_{2}, \cdots, X_{K}$, indicating that the detection order has no affect on the sum capacity. For this reason, in the remainder of the paper, it is assumed that the receiver is equipped with a SIC ML decoder and that the user data is detected in the descending order of channel gains.

\subsection{Broadcast Channel}

Figure 2 is the system model for the multi-user BC system working in superposition code strategy [2]. The data of users is independently encoded at the base station and then the combined signal is broadcast to all users. The channel for user $j$ can be considered as a MAC with an equal channel gain $g_{j}$ and the signal received at user $j$ can be written as

$$
Y_{j}=\sqrt{g_{j}} \sum_{i=1}^{K} \sqrt{P_{i}} X_{i}+Z_{j}
$$

where $Z_{j} \sim \mathcal{C N}(0,1)$ for $j=1,2, \cdots, K$ are i.i.d. complex Gaussian noise, $X_{i}$ is the transmitted signals of user $i, P_{i}$ is the power allocated to user $i, g_{j}$ is the channel gain from base station to user $j$. We also assume that $0<g_{1} \leq g_{2} \leq \cdots \leq g_{K}$.

The achievable rate $R_{j}$ is upper bounded by the capacity of the $j$-th link which can be written as

$$
C_{j}\left(\gamma_{j}\right)=I\left(Y_{j} ; X_{j}\right)
$$

The mutual information is monotonically increase with channel gain, so it is obvious that

$$
I\left(Y_{j+1} ; X_{j} \mid X_{j-1} \cdots X_{1}\right) \geq I\left(Y_{j} ; X_{j} \mid X_{j-1} \cdots X_{1}\right)
$$


which means that, if user $j$ can successfully decode its data $X_{j}$, so can user $j+1$ who has a better channel for $g_{j+1} \geq g_{j}$. Under the superposition coding strategy, user 1 treats the signals of other users as noise and decodes its data $X_{1}$ from $Y_{1}$. Then, user 2, which has the better channel can first decode $X_{1}$ of user 1 from $Y_{2}$ and proceed to subtract the transmit signal of user 1 from $Y_{2}$ and decode its data $X_{2}$ and so on.

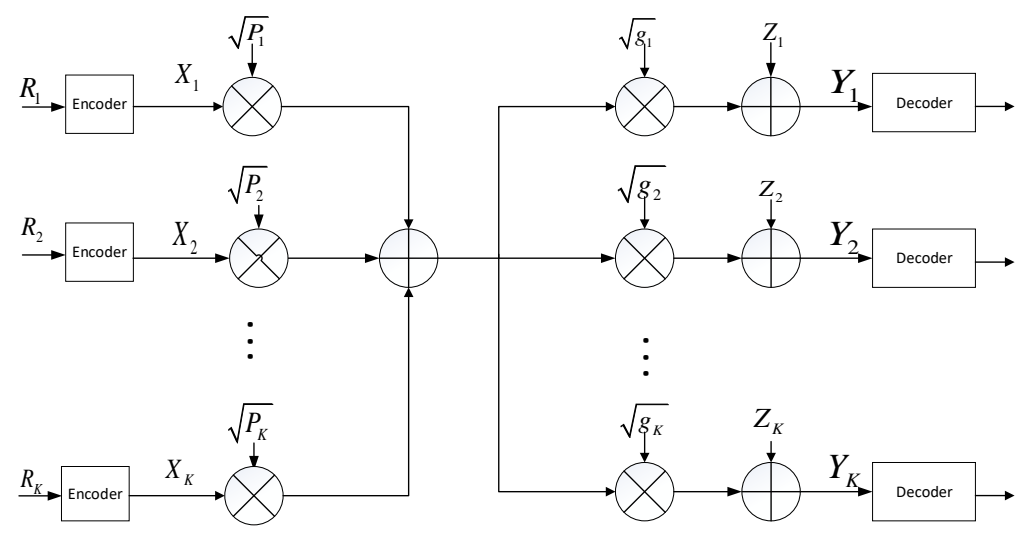

Figure 2. System model for a multi-user BC system.

For Gaussian channel inputs, the sum capacity for $\mathrm{BC}$ under a given power allocation $P_{1}, P_{2}, \cdots, P_{K}$ can be expressed [2] as

$$
\begin{aligned}
C_{\text {sum }}^{\text {Gaussian }} & =\sum_{j=1}^{K} I\left(Y_{j} ; X_{j}\right) \\
& =\sum_{j=1}^{K} \ln \left(1+\frac{P_{j} g_{j}}{1+g_{j} \sum_{i=j+1}^{K} P_{i}}\right) \\
& =\ln \left(1+\sum_{j=1}^{K} P_{j} g_{j}\right),
\end{aligned}
$$

which can be maximized by allocating total power $P_{1}+P_{2}+\cdots+P_{K}=P$ to the best user and the resulting maximum sum capacity is

$$
C_{\text {sum }}^{\text {Gaussian }}=\ln \left(1+P g_{K}\right) .
$$

Note that (14) and (15) are exactly the same as (3) and (4). This property is known as the duality of Gaussian BC and MAC [2].

For non-Gaussian inputs, the sum capacity for $\mathrm{BC}$ is given by

$$
\begin{aligned}
C_{\text {sum }} & =\sum_{j=1}^{K} I\left(Y_{j} ; X_{j}\right) \\
& =I\left(Y_{1} ; X_{1}\right)+I\left(Y_{2} ; X_{2} \mid X_{1}\right)+\cdots+I\left(Y_{K} ; X_{K} \mid X_{K-1} \cdots X_{1}\right) .
\end{aligned}
$$

\section{Entropy Power and the Parallelization of MAC and BC}

For Gaussian inputs, due to the very simple form of $\ln (1+\gamma)$, it is trivial to calculate the sum capacity and to find the optimal power allocation. However, this is far more complicated for non-Gaussian inputs since the integral in the mutual information calculation involves the PDF of $Y$ 
which is a combination of many Gaussian PDFs. This section will first introduce the concept of entropy power approximation and then convert the $K$ user MAC and BC into $K$ parallel point-to-point channels.

\subsection{Entropy Power}

Let $Z \sim \mathcal{C N}\left(0, \sigma_{Z}^{2}\right), X$ be an arbitrary random variable with zero mean and variance $\mathbb{E}\left[|X|^{2}\right]=\sigma_{X}^{2}$. $Z$ and $X$ are mutually independent. Assume that the mutual information $I(X ; X+Z)$ is known. Now we consider to approximate $X+Z$ as a Gaussian variable $\tilde{Z} \sim \mathcal{C} \mathcal{N}\left(0, \sigma_{\mathrm{e}}^{2}\right)$ while keeping entropy unchanged, i.e., $h(X+Z)=h(\tilde{Z})$.

Since $h(Z)=\ln \left(2 \pi \mathrm{e} \sigma_{Z}^{2}\right), h(\tilde{Z})=\ln \left(2 \pi \mathrm{e} \sigma_{\mathrm{e}}^{2}\right)$, and $I(X ; X+Z)=h(X+Z)-H(Z)$, so it is obvious that

$$
\sigma_{\mathrm{e}}^{2}=\mathrm{e}^{I(X ; X+Z)} \sigma_{Z}^{2}
$$

and we have the following inequality

$$
\sigma_{Z}^{2} \leq \sigma_{\mathrm{e}}^{2} \leq \sigma_{X}^{2}+\sigma_{Z}^{2}
$$

due to the fact that $0 \leq I(X ; X+Z) \leq \ln \left(1+\sigma_{X}^{2} / \sigma_{Z}^{2}\right) . \sigma_{\mathrm{e}}^{2}$ is known as the entropy power $[20,21]$ which is originally proposed by Claude Shannon.

\subsection{Parallelization for $M A C$}

Consider the MAC system shown in Figure 1. Assume that the base station has adopted a SIC decoder which detects user data in the descending order of channel gains, i.e., $X_{K}$ is first detected taking $X_{1}, X_{2}, \cdots, X_{K-1}$ as interference, then the $X_{K-1}$ is detected with $X_{K}$ being cancelled out and the interference being $X_{1}, X_{2}, \cdots, X_{K-2}$, and so on.

In detecting the last data $X_{1}$, the equivalent received signal is

$$
Y_{1}=\sqrt{P_{1} g_{1}} X_{1}+Z
$$

The capacity of user 1 is given by

$$
C_{1}=C_{0}\left(\gamma_{1}\right)=C_{0}\left(P_{1} g_{1}\right),
$$

where $C_{0}(x)$ is the function defined in (8) and can be estimated with (9).

In detecting $X_{2}$, the equivalent received signal is

$$
Y_{2}=\sqrt{P_{2} g_{2}} X_{2}+\sqrt{P_{1} g_{1}} X_{1}+Z
$$

According to (17), the last two terms $\sqrt{P_{1} g_{1}} X_{1}+Z$ of (21) can be approximated to a Gaussian noise of entropy power $\sigma_{1}^{2}=\mathrm{e}^{-C_{1}}$. Scaling both sides of (21) by $\sigma_{1}$ yields

$$
\tilde{Y}_{2}=\sqrt{P_{2} \tilde{g}_{2}} X_{2}+Z_{2}
$$

where $\tilde{g}_{2}=g_{2} \mathrm{e}^{-C_{1}}$ and $Z_{2} \sim \mathcal{C N}(0,1)$ is the Gaussian approximation of $\left(\sqrt{P_{1} g_{1}} X_{1}+Z\right) / \sigma_{1}$. This equation indicates that, from the perspective of user 2, the multi-user MAC channel has been converted to a single user channel where the existence of interfering user 1 is equivalent to modify the channel gain $g_{2}$ by a factor $\mathrm{e}^{-C_{1}}$.

In the similar way, it is straightforward to see that the channel for user $j$ is equivalent to

$$
\tilde{Y}_{j}=\sqrt{P_{j} \tilde{g}_{j}} X_{j}+Z_{j}
$$


where $Z_{j} \sim \mathcal{C N}(0,1)$ is the normalized Gaussian approximation of interference and noise, and the equivalent channel gain is given by

$$
\begin{aligned}
\tilde{g}_{j} & =g_{j} \mathrm{e}^{-C_{1}-C_{2}-\cdots-C_{j-1}} \\
C_{j} & =C_{0}\left(P_{j} \tilde{g}_{j}\right)
\end{aligned}
$$

for $j=1,2, \cdots, K$ with $\tilde{g}_{1}=g_{1}$.

Now, (24) indicates that the $K$ users MAC system is equivalent to $K$ parallel single user systems each with the channel gain $g_{j}$ being modified to $\tilde{g}_{j}$, as shown in Figure 3 . The sum capacity is then given by

$$
C_{\text {sum }} \approx \sum_{j=1}^{K} C_{j}=\sum_{j=1}^{K} C_{0}\left(P_{j} \tilde{g}_{j}\right),
$$

which can be evaluated via (8) or (9).

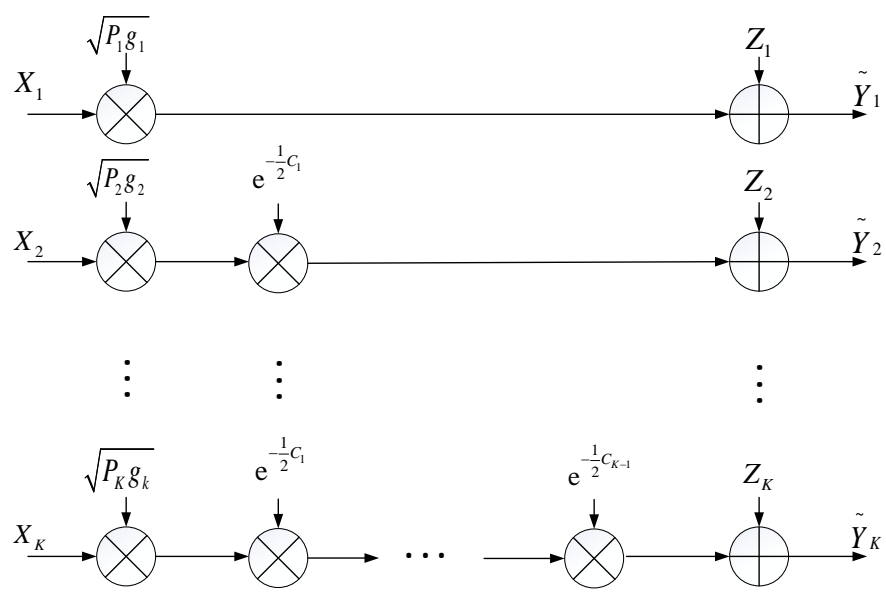

Figure 3. Parallelization for MAC.

Figure 3 indicates that the existence of interfering users is equivalent to an extra attenuation of channel gain. It can be noted that the capacity of $M$-ary modulation ranges from 0 to $\log M$, therefore it is obviously that

$$
g_{j} \geq \tilde{g}_{j} \geq \frac{g_{j}}{M^{j-1}}
$$

for $j=1,2, \cdots, K$. At low SNR regime, $\tilde{g}_{j}$ goes to $g_{j}$ while at high SNR regime $\tilde{g}_{j}$ goes to $g_{j} / M^{j-1}$.

\subsection{Parallelization for $B C$}

For the $K$ users BC system operated in superposition mode, user $j$ can cancel the interference from user 1 to $j-1$. The received signal after interference cancellation can be expressed as

$$
Y_{j}=\sqrt{P_{j} g_{j}} X_{j}+\sqrt{g_{j}} \sum_{i=j+1}^{K} \sqrt{P_{i}} X_{i}+Z_{j}
$$

Similar to the case in MAC, by approximating $Z^{\prime}=\sqrt{g_{j}} \sum_{i=j+1}^{K} \sqrt{P_{j}} X_{i}+Z_{j}$ to a Gaussian noise with equivalent noise variance be the entropy power of $Z^{\prime}$, and after noise power normalization, the channel form $X_{j}$ to user $j$ can be equivalent to

$$
\tilde{Y}_{j}=\sqrt{P_{j} \tilde{g}_{j}} X_{j}+\tilde{Z}_{j},
$$


where $\tilde{Z}_{j} \sim \mathcal{C N}(0,1)$ is the normalized effective Gaussian noise, $\tilde{g}_{j}$ is the equivalent channel gain given by

$$
\tilde{g}_{j}=g_{j} \mathrm{e}^{-\sum_{j=i+1}^{K} C_{j i}}
$$

with

$$
C_{j i}=I\left(Y_{j} ; X_{i}\right)
$$

be the mutual information between the transmitted data $X_{i}$ and the received signal $Y_{j}$ which can be evaluated by regarding the channel between $X_{j}, X_{j+1}, \cdots, X_{K}$ to $Y_{j}$ as a MAC but with inverse SIC detection order.

In such a way, the $K$ user BC system is also equivalent to $K$ parallel point-to-point channels as depicted in Figure 4.
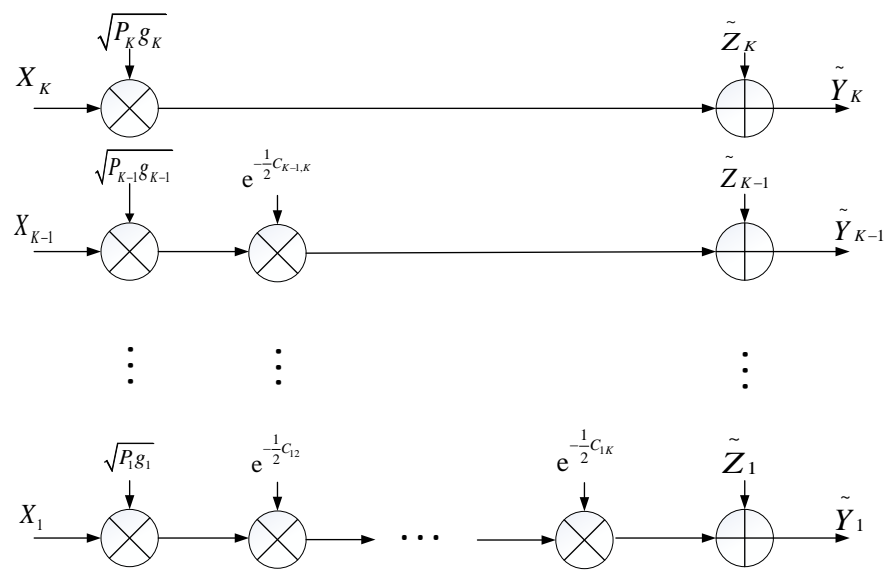

Figure 4. Parallelization for BC.

As Figure 4 has the same structure as Figure 3 with the only difference lying in the scaling coefficient between $g_{j}$ and $\tilde{g}_{j}$, we will only consider MAC hereafter.

\section{Sum Capacity for MAC with Different Power Allocation Strategies}

Based on the parallel model shown in Figure 3, this section will discuss the capacity of MAC channel under different power allocation strategies.

\subsection{Equal Power Distribution}

In the case of equal power allocation, the total transmit power $P$ is uniformly allocated among $K$ users, i.e., $P_{1}=P_{2}=\cdots=P_{K}=\frac{P}{K}$. The signal arrived at the base station is

$$
Y=\sqrt{\frac{P}{K}} \sum_{j=1}^{K} \sqrt{g_{j}} X_{j}+Z
$$

and the sum capacity is given by

$$
C_{\text {sum }}=\sum_{j=1}^{K} C_{j}=\sum_{j=1}^{K} C_{0}\left(\frac{P \tilde{g}_{j}}{K}\right)
$$

where $\tilde{g}_{j}=g_{j} \cdot \mathrm{e}^{-C_{1}-C_{2}-\cdots-C_{j-1}}$ is the scaled equivalent channel gain, and $C_{0}(\cdot)$ can be evaluated with the approximation Formula (9). 
It can be noted that the sum capacity given by (32) is upper bounded by

$$
C_{\text {sum }} \leq \sum_{i=1}^{K} \log \left(1+\frac{P g_{i}}{K}\right)
$$

since the Gaussian encoder has maximum channel mutual information. In addition, when $K \rightarrow \infty$, the sum capacity with $M$-ary inputs will approach to the upper bound because that the term $\frac{1}{\sqrt{K}} \sum_{j=1}^{K} \sqrt{g_{j}} X_{j}$ in (31) will approach to Gaussian according to central limit theorem (CLT). Asymptotically, $\lim _{K \rightarrow \infty} \frac{1}{\sqrt{K}} \sum_{j=1}^{K} \sqrt{g_{j}} X_{j} \sim \mathcal{C N}(0,1)$ with $\mathbb{E}\left[g_{i}\right]=1$ and the capacity will go to $\ln (1+P)$. In other words, the Shannon capacity can be realized with a large number of small power nodes, no matter what the constellation size is.

\subsection{Power Allocation for Equal Capacity}

If the objective is to guarantee all users have the same quality-of-service (QoS), i.e., $C_{1}=C_{2}=\cdots=C_{K}=C$, then from Figure 3 it is obvious that the power allocation should satisfy

$$
P_{i} \tilde{g}_{i}=P_{i-1} \tilde{g}_{i-1}
$$

for all $i=2,3, \cdots, K$.

Equation (34) can be rewritten as

$$
P_{i}=P_{i-1} \cdot \frac{\tilde{g}_{i-1}}{g_{i}}=P_{i-1} \cdot \frac{g_{i-1}}{g_{i}} \mathrm{e}^{\mathrm{C}} .
$$

Apply (35) recursively, the result obtained is

$$
P_{i}=P_{1} \cdot \frac{g_{1}}{g_{i}} \mathrm{e}^{(i-1) C} .
$$

Whenever $P_{1}$ is given, $C=C_{0}\left(P_{1} g_{1}\right)$ and $P_{2}, P_{3}, \cdots, P_{K}$ are also given. Therefore, the optimal power allocation which can maximize the common capacity $C$ can be easily obtained by using numerical search algorithms, say, the dichotomy algorithm.

\subsection{Optimal Power Allocation for Maximum Sum Capacity}

This section focus on the optimal power allocation that can maximum the sum capacity of a $K$ user MAC system under the total power constraint. For the Gaussian inputs, the optimal solution is known as to allocate all power to the strongest user. However, this is not the case for $M$-ary inputs as will be seen latter.

The optimization problem can be formulated as

$$
\begin{array}{ll}
\text { maximize } & f\left(P_{1}, \cdots, P_{K}\right)=\sum_{j=1}^{K} C_{j}=\sum_{i=1}^{K} C_{0}\left(P_{i} \tilde{g}_{i}\right) \\
\text { subject to } & \sum_{j=1}^{K} P_{j}=P
\end{array}
$$

Define

$$
J=\sum_{i=1}^{K} C_{i}-\lambda\left(\sum_{i=1}^{K} P_{i}-P\right)
$$


If $\boldsymbol{P}^{*}=\left[P_{1}^{*}, P_{2}^{*}, \cdots, P_{K}^{*}\right]$ is the optimal solution for (37), then at $\boldsymbol{P}^{*}$, the partial derivatives of $f$ with respect to $P_{k}$ must satisfy

$$
\sum_{i=1}^{K} \frac{\partial C_{i}}{\partial P_{k}}=\lambda, \quad k=1,2, \cdots, K
$$

Define

$$
\lambda_{k}=\sum_{i=1}^{K} \frac{\partial C_{0}\left(P_{i} \tilde{g}_{i}\right)}{\partial P_{k}}
$$

which represents the slop of sum capacity with respect $P_{k}$, then the necessary condition for the optimal power allocation can be stated as

$$
\lambda_{1}=\lambda_{2}=\cdots=\lambda_{K}
$$

Based on this necessary condition, an iterative algorithm for the optimal power allocation is shown in Algorithm 1.

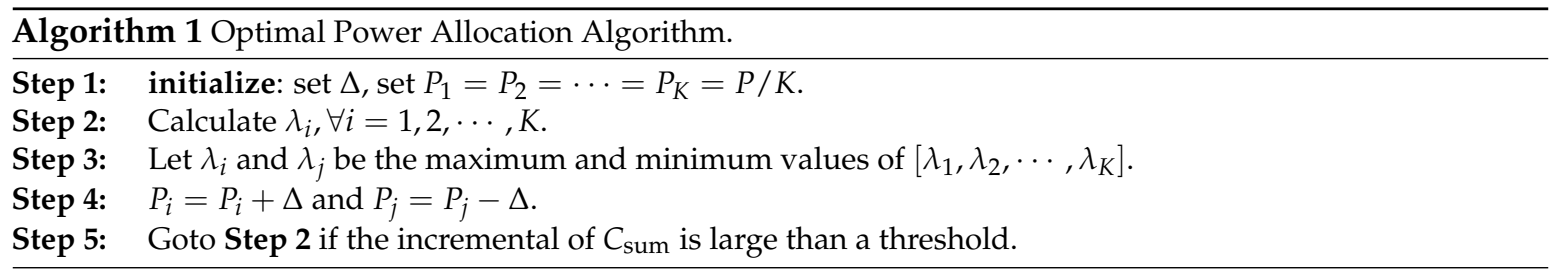

It can be observed from (26) that, at low SNR regime, $\tilde{g}_{j}$ will go to $g_{j}$. In this situation, Figure 3 becomes $K$ parallel independent channels without interaction among channel gains. According to [22], the optimal power allocation is to allocate power only to the strongest channel and the sum capacity is simply given by $C_{0}\left(P g_{K}\right)$.

At high SNR regime, by applying the lower bound in (26)-(37), the optimization problem becomes

$$
\begin{array}{ll}
\text { maximum } & f\left(P_{1}, \cdots, P_{K}\right)=\sum_{j=1}^{K} C_{0}\left(\frac{P_{j} g_{j}}{M^{j-1}}\right) . \\
\text { subject to } & \sum_{j=1}^{K} P_{j}=P \rightarrow \infty
\end{array}
$$

It can be verified that the objective function $f\left(P_{1}, \cdots, P_{K}\right)$ is concave. According to [22], the optimal power allocation is given by

$$
P_{j}=\frac{\beta}{\tilde{g}_{j}} P
$$

with

$$
\frac{1}{\beta}=\frac{1}{K} \sum_{i=1}^{K} \frac{1}{\tilde{g}_{j}}
$$

This indicates that the stronger an equivalent channel is, the less power it is allocated which is in sharp contrast to water-filling policy. The sum capacity for this case is given by

$$
C_{\text {sum }}=\sum_{i=1}^{K} C_{0}(\beta P)
$$


Equations (39) and (43) rely on the Karush-Kuhn-Tucker (KKT) conditions [23]. In general, there is a unique $P^{*}$ that satisfies the KKT conditions when problem (37) is strictly concave, corresponding to the global maximum. However, the optimal power allocation in (37) is nonconcave. It becomes concave only in some specific cases, e.g., for Gaussian input distributions (4). Especially, (37) is also concave at low and high SNR regime in which case the $K$ parallel channels are independent, i.e., the power allocation (43) is unique. However, in general, (39) does not identify uniquely the optimal power allocation. Thus, it is important to realize that (39) gives a necessary condition for the optimal power allocation.

\section{Numerical Results}

This section presents the numerical results of the sum capacity for single-cell multi-user system. Since Figure 4 is essentially the same with Figure 3, only the results of MAC are presented. Throughout this section, $\left\{g_{j}\right\}$ are i.i.d. exponentially distributed random variables with unit expectation, i.e., $\mathbb{E}\left[g_{j}\right]=1$ which means the data of each user $X_{i}$ goes through Rayleigh fading and SNR represents the average signal-to-noise ratio per user. The true quantities are approximated using standard Monte Carlo techniques for comparison.

Figure 5 depicts the capacity calculated by (9) versus average SNR per user. It can be seen that the curves obtained by (9) for QPSK, 8PSK, 16QAM modulations fit well with the exact capacity which is obtained with Monte Carlo integration [18].

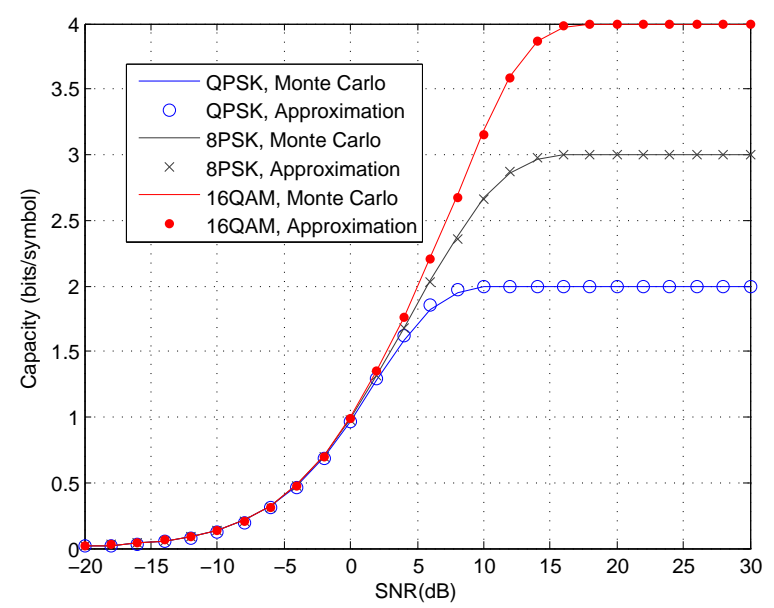

Figure 5. Approximations for the capacity with QPSK, 8PSK, 16QAM modulations, respectively.

Figure 6 evaluates the sum capacity with the parallel model shown in Figure 3 . The results of (25) with 16QAM modulation and $K=2,3,4,10$ are illustrated respectively, assuming the total power $P$ is randomly allocated to users. The abscissa is $P$ in decibel and the unit of capacity is bits per symbol. The curves labeled by "Monte Carlo, $K=2,3$ " are obtained via the Monte Carlo integration of (5) and the other curves are evaluated from (25). It can be observed that the approximations fit well with the result of Monte Carlo integration when SNR is small and has a small error with the real values when SNR is pretty large which means that the approximations can be considered as a good alternative for the sum capacity. Note that the curves "Monte Carlo, $K=4,10$ " are missing because it is almost impossible to use Monte Carlo integration for estimating these cases since $p(y)$ defined in (6) has $16^{4}$ and $16^{10}$ items in summation. The cases of $K=6,8$ and so on are similar to the cases of $K=2,3,4,10$. 


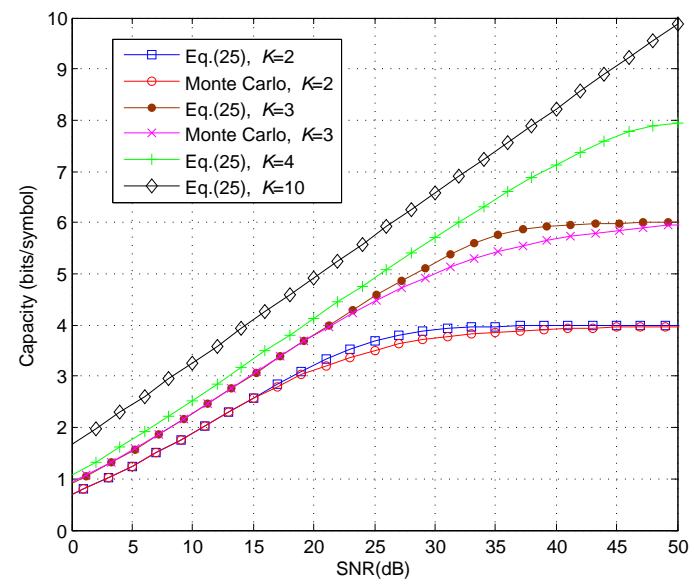

Figure 6. Approximations for the sum capacity with $K=2,4,10$, respectively.

Figures 7 and 8 give the sum capacity obtained by equal power allocation, equal capacity power allocation and optimal maximum capacity power allocation for MAC with 16QAM modulation and $K=2,4$ users, respectively. The sum capacity is always upper limited by $K \log M$ for all strategies. The gap between the sum capacity of optimal power allocation and that of equal power allocation first becomes larger with the increase of total power and then gets to the upper limit. As for the equal capacity power allocation, the gap to the maximum capacity is relatively large when the total power is small because the optimal power allocation is to allocate power only to the strongest channel.

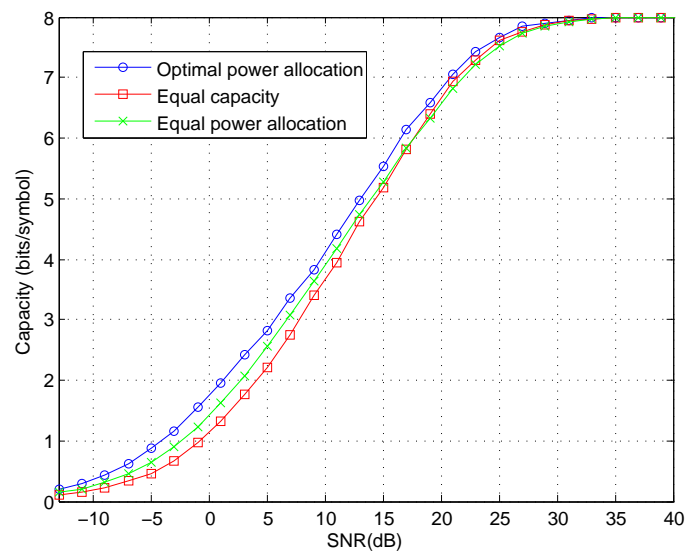

Figure 7. Sum capacity for 2 users MAC with 16QAM.

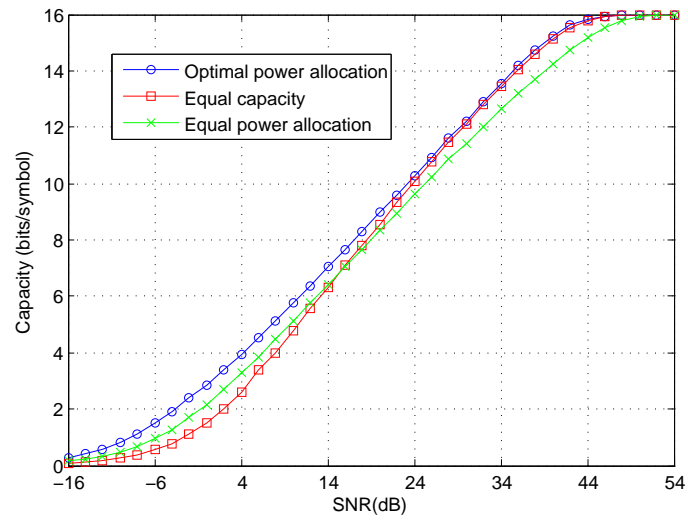

Figure 8. Sum capacity for 4 users MAC with 16QAM. 
Figures 9 and 10 indicate that the increment of sum capacity with different power allocation strategies becomes smaller with the increase of users $K$ when $P=0 \mathrm{~dB}, 10 \mathrm{~dB}$ and $30 \mathrm{~dB}$, respectively. The optimal power allocation performs the best and the sum capacities with equal power allocation strategy are upper bounded by $\log _{2}(1+P)$ as shown in Section 4.1. The gap between equal capacity power allocation and optimal power allocation becomes larger as the number of user $K$ increases because each user can reach the upper limit of the channel capacity $\log M$ when $K$ is small and $P$ is relatively large, i.e., the optimal power allocation strategy is equivalent to equal capacity power allocation in this case. It can be observed that the sum capacity of equal capacity power allocation decreases as the number of user increases when $P$ is small due to the fact that the optimal power allocation is to allocate power only to the strongest channel with low SNR regime.

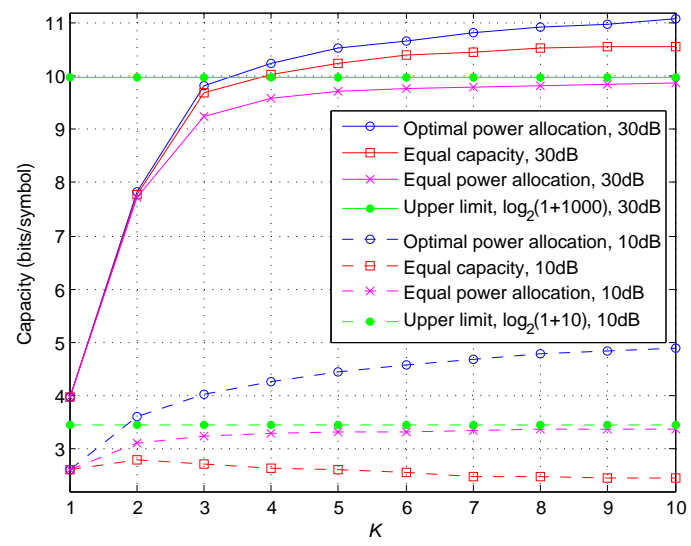

Figure 9. Sum capacity with total power $P=10 \mathrm{~dB}$ and $30 \mathrm{~dB}$.

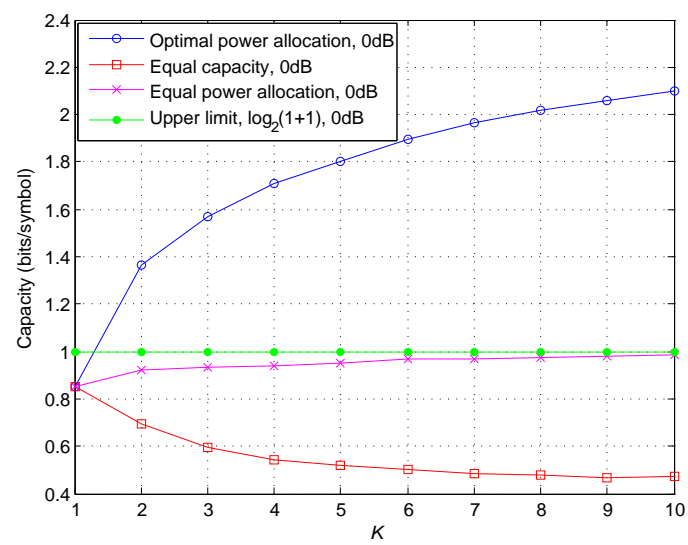

Figure 10. Sum capacity with total power $P=0 \mathrm{~dB}$.

Figures 11-13 illustrate the optimal power allocation and equal capacity power allocation with total power $P=-3,30,50 \mathrm{~dB}$, respectively. The channel gains for the 4 users are $g=[0.2309,0.5543,0.9139,1.5171]$. When the total power is small, i.e., $-3 \mathrm{~dB}$, the equivalent channel gains are the same with real channel gains $g$, the optimal power allocation is to allocate power only to the strongest channel which is consistent with the power allocation for Gaussian MAC and BC. Observed from Figure 11, the channel gain of the 4th user is the best, thus all the power is allocated to user 4 and the power for other users is zero. In addition, to achieve the equal capacity, the power allocation for each user is inversely proportional to its channel gain which is in shape contrast with optimal power allocation. Figure 12 shows the power allocation with total power $P=30 \mathrm{~dB}$, the difference between equal capacity and optimal power allocation becomes small compared with Figure 11 which can also be obtained from Figure 8. Figure 13 illustrates the power distribution 
with $K=4$ and total power $P=50 \mathrm{~dB}$. When $P$ is large, first we can get an equivalent channel gain through (24), and the better the equivalent channel gain is, the less power it is allocated. In this case, the difference between the optimal and equal power allocation is small because they can both achieve the upper bound of the capacity, thus the equivalent channel gains (24) for the two strategies have no difference.

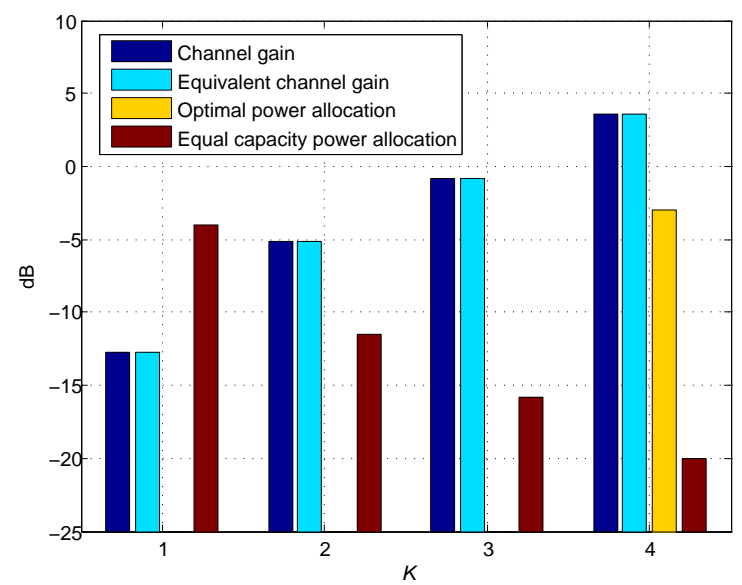

Figure 11. Power allocation with total power $P=-3 \mathrm{~dB}$.

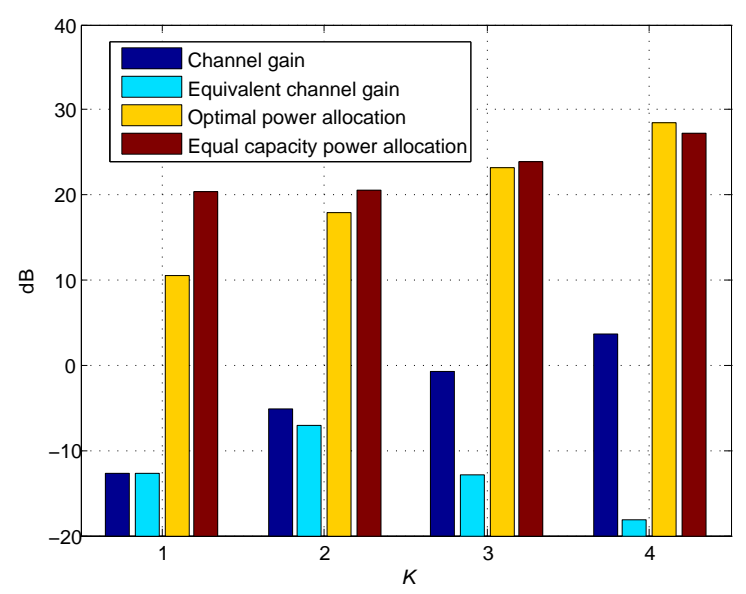

Figure 12. Power allocation with total power $P=30 \mathrm{~dB}$.

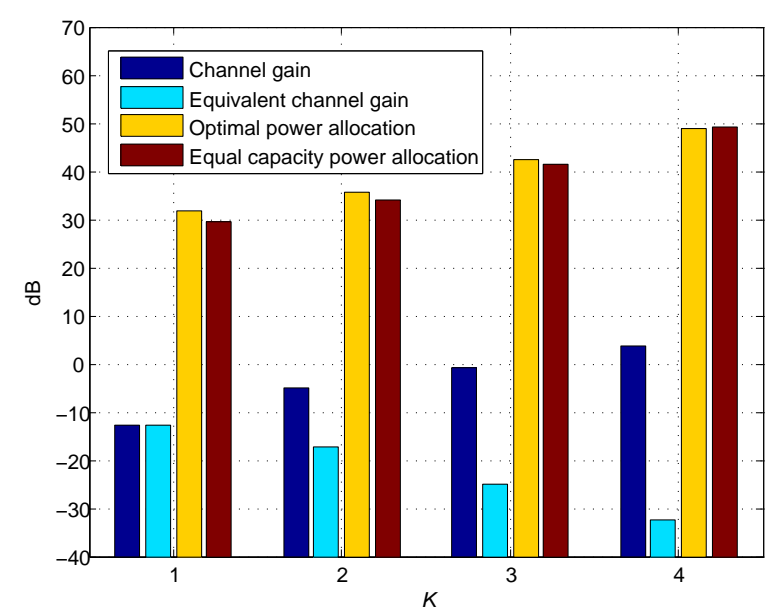

Figure 13. Power allocation with total power $P=50 \mathrm{~dB}$. 


\section{Conclusions}

This paper first introduces an approximation which gives a good result for the capacity of point-to-point communication with arbitrary inputs AWGN channel. For multi-user systems, it is shown that both MAC and BC can be approximated to a bank of parallel channels based on SIC and entropy power Gaussian approximation. Then the sum capacity for MAC and BC can be approximated to the sum of capacity of $K$ parallel channels with arbitrary inputs.

Based on the approximation and the parallelization of MAC and BC, an optimal power allocation algorithm that maximizes the sum capacity with non-Gaussian inputs is proposed by (39). When SNR is large, e.g., $50 \mathrm{~dB}$, the optimal power allocation is to allocate its power to a series of equivalent channels and the stronger the equivalent channel is, the smaller the power of the related channel is allocated. When SNR is small, e.g., $-3 \mathrm{~dB}$, the optimal power allocation is independent of the channel inputs, i.e., the optimal power allocation is to allocate all the power only to the strongest channel whether it is Gaussian or non-Gaussian. For the equal capacity power allocation, a recursive relation for the solution of power allocation is derived.

Future work will focus on multiuser channels with multiple antennas in both the uplink and the downlink. With the increase of the degrees of freedom from having multiple antennas, the gain of the sum capacity will be investigated.

Acknowledgments: This work was supported by the Ministry of Education and China Mobile Joint Scientific Research Fund (Grant No. MCM20160105).

Author Contributions: Pei Yang and Hongwen Yang designed the experiments and wrote the paper; Yue Wu contributed the simulation tools; Liqiang Jin and Hongwen Yang made the revisions for the paper; All authors have read and approved the final manuscript.

Conflicts of Interest: The authors declare no conflict of interest.

\section{References}

1. Jindal, N.; Vishwanath, S.; Goldsmith, A. On the duality of Gaussian multiple-access and broadcast channels. IEEE Trans. Inf. Theory 2004, 50, 768-783.

2. Goldsmith, A. Multiuser systems. In Wireless Communications; Cambridge University Press: Cambridge, UK, 2005; pp. 297-314.

3. Tse, D.; Viswanath, P. Multiuser capacity and opportunistic communication. In Fundamentals of Wireless Communication; Cambridge University Press: Cambridge, UK, 2005; pp. 228-278.

4. Cover, T.M.; Thomas, J.A. Multiuser Information Theory. In Elements of Information Theory; Wiley: New York, NY, USA, 2012; pp. 509-609.

5. Benfarah, A.; Tomasin, S.; Laurenti, N. Power Allocation in Multiuser Parallel Gaussian Broadcast Channels With Common and Confidential Messages. IEEE Trans. Commun. 2016, 64, 2326-2339.

6. Yang, S.; Jiang, T. Closed-Form Optimal Power Allocation for Weighted Rate Sum Maximization in Gaussian Broadcast Channel. IEEE Trans. Commun. 2012, 60, 1782-1787.

7. Jiang, W.; Cui, Y. Comments on "Closed-Form Optimal Power Allocation for Weighted Rate Sum Maximization in Gaussian Broadcast Channel". IEEE Trans. Commun. 2014, 62, 316-319.

8. He, P.; Zhao, L. Optimal Power Allocation for Maximum Throughput of General MU-MIMO Multiple Access Channels with Mixed Constraints. IEEE Trans. Commun. 2016, 64, 1042-1054.

9. Liang, J.; Wang, X.; Zhang, W. Capacity Theorem and Optimal Power Allocation for Frequency Division Multiple-Access Relay Channels. In Proceedings of the IEEE Global Telecommunications Conference (GLOBECOM), Kathmandu, Nepal, 5-9 December 2011.

10. Kang, X. Optimal Power Allocation for Fading Cognitive Multiple Access Channels: A Two-User Case. IEEE Wirel. Commun. Lett. 2013, 6, 683-686.

11. Fong, S.L.; Tan, V.Y.F. A Proof of the Strong Converse Theorem for Gaussian Multiple Access Channels. IEEE Trans. Inf. Theory 2016, 62, 4376-4394.

12. Romero, H.P.; Varanasi, M.K. The K-User Vector Gaussian Multiple-Access Channel With General Messages Sets: Capacity, Polymatroidal Structure, and Efficient Computation. IEEE Trans. Inf. Theory 2017, 63, 3875-3893. 
13. Lin, L.; Ma, X.; Liang, C.; Huang, X.; Bai, B. An Information-Spectrum Approach to the Capacity Region of the Interference Channel. Entropy 2017, 19, 270.

14. Baccarelli, E. Asymptotically tight bounds on the capacity and outage probability for QAM transmissions over Rayleigh-faded data channels with CSI. IEEE Trans. Commun. 1999, 47, 1273-1277.

15. Slimane, S.B. Approximation to the symmetric capacity of Rayleigh fading channels with multi-level signals. IEEE Commun. Lett. 2006, 10, 129-131.

16. Yang, P.; Wu, Y.; Yang, H. Capacity of Nakagami-m Fading Channel With BPSK/QPSK Modulations. IEEE Commun. Lett. 2017, 21, 564-567.

17. Yang, P.; Jin, L.; Yang, H. An improved approximation formula for the symmetric capacity of 2-dimentional constellations under AWGN channel. In Proceedings of the 2016 International Conference on Computer Science, Technology and Application (CSTA2016), Changsha, China, 2016; World Scientific: Singapore, 2016.

18. Ungerboeck, G. Channel coding with multilevel/phase signals. IEEE Trans. Inf. Theory 1982, $28,55-67$.

19. Rioul, O. Information theoretic proofs of entropy power inequalities. IEEE Trans. Inf. Theory 2011, 57, 33-55.

20. Verdú, S.; Guo, D. A simple proof of the entropy-power inequality. IEEE Trans. Inf. Theory 2006, 52, $2165-2166$.

21. Shannon, C.E; Weaver, W. A mathematical theory of communication. Bell Syst. Tech. J. 1948, 27, 379-423.

22. Lozano, A.; Tulino, A.M.; Verdú, S. Optimum power allocation for parallel Gaussian channels with arbitrary input distributions. IEEE Trans. Inf. Theory 2006, 52, 3033-3051.

23. Fletcher, R. The Theory of Constrained Optimization. In Practical Methods of Optimization; Wiley: New York, NY, USA, 1987; pp. 195-224.

(C) 2017 by the authors. Licensee MDPI, Basel, Switzerland. This article is an open access article distributed under the terms and conditions of the Creative Commons Attribution (CC BY) license (http://creativecommons.org/licenses/by/4.0/). 